\title{
Rendezvous of Retro2 at the ER
}

\author{
Hesso Farhan
}

Retro-2 is a drug that protects cells against bacterial and plant toxins by arresting their trafficking in endosomes. New data show that the target for Retro- 2 is surprisingly not within the endosomal system, but on the endoplasmic reticulum.

Many bacterial and plant toxins undertake a journey starting from the plasma membrane, going over several endomembrane organelles to finally reach the endoplasmic reticulum (ER). For instance, the Shiga toxin and Shiga-like toxins (SLT), once internalized, travel from early endosomes towards the Golgi and then traffic back to the ER. Various factors have been identified to mediate transit of Shiga toxin from endosome to the ER. For example, GPP130 is a manganese-sensitive membrane protein is known to be involved in trafficking of Shiga toxins from endosomes to the Golgi ${ }^{1}$. Understanding the various retrograde trafficking pathways may enable the identification of novel drug targets. The small molecule Retro-2 was known to block SLT trafficking between the endosomes and the Golgi. Although the target of Retro-2 was predicted to be in the endosomal system, the mechanism of action remained elusive. In this issue, Forrester and colleagues ${ }^{2}$ identified the target of Retro- 2 as Sec16A, a protein known to act as a scaffold that organizes ER exit sites (ERES)

ERES are specialized domains of the rough ER that act as nucleation sites for the formation of COPII carriers. Sec16A is essential for the biogenesis and maintenance of ERES in mammalian cells and acts as an integrator for signaling pathways to $\mathrm{ERES}^{3-5}$. Sec16A binds to all COPII components and promotes budding of COPII carriers from the ER. The current work shows that Retro-2 reduces the interaction of COPII components with Sec16A, suggesting an effect on ER export. The COPII coat captures cargo via its Sec24 subunit. For example, syntaxin 5 binds to Sec24C\&D isoforms and is thereby concentrated into COPII vesicles ${ }^{6}$. Synatxin 5 is a SNARE protein that plays a role in membrane trafficking between the ER and the Golgi ${ }^{7}$. In line with its effect on Sec16A-COPII coupling, Retro-2 inhibited the export of syntaxin 5 from the ER, which explains the previous observation that this drug results in redistribution of synatxin 5 to the $\mathrm{ER}^{8}$. Synatxin 5 is not known to localize to the endosomal system, so how does its ER retention lead to a trafficking defect of Shiga toxin from endosomes to the Golgi? The hypothesis was tested whether Retro-2 retains syntaxin 5 in the ER, and thereby prevents it from meeting a Golgilocalized partner required for Shiga toxin trafficking. Forrester et $\mathrm{al}^{2}$ identified GPP130 (also known as GOLIM) as a novel interaction partner for syntaxin 5 . The interaction with syntaxin 5 appears to be important for the localization of GPP130 to the Golgi, because a mutant of GPP130 deficient in syntaxin 5 binding, exhibited a stronger endosomal localization. 
The mode of action proposed for Retro- 2 is as follows: Retro-2 binds to Sec16A and thereby inhibits ER export of synatxin 5, preventing it from encountering GPP130. Therefore, GPP130 is trapped in the endosomal system, which explains why Retro-2 inhibits transport of Shiga toxins from endosomes to the Golgi (Figure 1). As with any good paper, while it answers unresolved matters, it opens up new questions that need to be addressed in future studies. For instance, it is difficult to understand why Retro2 would specifically affect only trafficking of synatxin 5, because Sec16A is a general regulator of ER export and ERES homeostasis. Thus, we would expect that Retro-2 would have a more general effect on membrane trafficking and COPII at ERES. However, this is not the case. Retro-2 had no effect on the trafficking of any other secretory cargo out of the ER and it had no effect on the dynamics of COPII components on ERES or on the organization of these ER subdomains. Sec16A is a multidomain protein of $250 \mathrm{kDa}$ that is heavily post-translationally modified (www.phosphosite.org/). Thus, it is possible that different states, or pools of Sec16A exist and that Retro-2 selectively affects one of these. Clearly, more work is needed to resolve these details.

Besides uncovering the mechanistic target of Retro-2, the current work has another important impact on the field of chemical biology. Chemical biologists traditionally considered ER export notoriously undruggable for two reasons: firstly, the components of this machinery are evolutionarily conserved and considered essential for cellular homeostasis. Secondly, it is generally assumed that the ER export machinery acts constitutively and that its components are active on all ERES. Thus, targeting any component is likely to produce generalized pleotropic effects on trafficking. The work of Forrester and colleagues $^{2}$ shows that targeting ER export may be useful to block bacterial toxin trafficking. Taken together with the recent discovery of ER export regulating kinases ${ }^{9,10}$, the community may now probe the role of these kinases in the trafficking of bacterial and plant toxins. Altogether, this new work identifies Sec16A as an unexpected target for Retro-2 and opens possibilities for future investigations on the possibility to target components of the ER export machinery to alter only a discrete subset of its function, and avoiding pleotropic effects.

Hesso Farhan is at the Institute of Basic Medical Sciences, Department of Molecular Medicine, University of Oslo, Norway

E-mail: hesso.farhan@medisin.uio.no

Figure 1. The newly identified mode of action of Retro2. Left sides: Sec16A-dependent trafficking of syntaxin 5 to the Golgi, where it encounters GPP130. Shiga toxin enters cells by endocytosis and required GPP130 to reach the Golgi. Syntaxin 5 plays a role in regulating Shiga toxin trafficking, by regulating endosome-Golgi cycling of GPP130. Right side: when cells are treated with Retro-2, Sec16A binds less to COPII resulting in reduced trafficking of syntaxin 5 to the Golgi. Consequently, GPP130 (together with Shiga toxin) is trapped in endosomes and fails to reach the Golgi. 


\section{References}

1 Natarajan, R. \& Linstedt, A. D. A cycling cis-Golgi protein mediates endosome-to-Golgi traffic. Mol Biol Cell 15, 4798-4806, doi:10.1091/mbc.e04-05-0366 (2004).

2 Alison Forrester, S. J. R., Maria Daniela Garcia-Castillo, Collin Bachert, Audrey Couhert, Livia Tepshi, Sylvain Pichard, Jennifer Martinez, Mathilde Munier, Raphael Sierocki, Henri-François Renard, César Augusto Valades-Cruz, Florent Dingli, Damarys Loew, Christophe Lamaze, Jean-Christophe Cintrat, Adam D. Linstedt, Daniel Gillet, Julien Barbier, Ludger Johannes. FUNCTIONAL DISSECTION OF THE RETROGRADE SHIGA TOXIN TRAFFICKING INHIBITOR RETRO-2. Nature Chemical Biology (2020).

3 Hughes, H. et al. Organisation of human ER-exit sites: requirements for the localisation of Sec16 to transitional ER. J Cell Sci 122, 2924-2934, doi:10.1242/jcs.044032 (2009).

4 Farhan, $\mathrm{H}$. et al. MAPK signaling to the early secretory pathway revealed by kinase/phosphatase functional screening. J Cell Biol 189, 997-1011, doi:10.1083/jcb.200912082 (2010).

5 Bhattacharyya, D. \& Glick, B. S. Two mammalian Sec16 homologues have nonredundant functions in endoplasmic reticulum (ER) export and transitional ER organization. Mol Biol Cell 18, 839-849, doi:10.1091/mbc.e06-08-0707 (2007).

6 Mancias, J. D. \& Goldberg, J. Structural basis of cargo membrane protein discrimination by the human COPII coat machinery. EMBO J 27, 2918-2928, doi:10.1038/emboj.2008.208 (2008).

7 Linders, P. T., Horst, C. v. d., Beest, M. T. \& van den Bogaart, G. Stx5-Mediated ER-Golgi Transport in Mammals and Yeast. Cells 8, 780, doi:10.3390/cells8080780 (2019).

8 Stechmann, B. et al. Inhibition of retrograde transport protects mice from lethal ricin challenge. Cell 141, 231-242, doi:10.1016/j.cell.2010.01.043 (2010).

9 Centonze, F. G. et al. LTK is an ER-resident receptor tyrosine kinase that regulates secretion. J Cell Biol 218, 2470-2480, doi:10.1083/jcb.201903068 (2019).

10 Subramanian, A. et al. Auto-regulation of Secretory Flux by Sensing and Responding to the Folded Cargo Protein Load in the Endoplasmic Reticulum. Cell 176, 14611476.e1423, doi:10.1016/j.cell.2019.01.035 (2019). 